\section{Potential for serodiagnosis of indonesian leprosy patients by detecting antibodies against LID-1}

\author{
Nanny Herwanto, ${ }^{1}$ Sawitri, ${ }^{1}$ \\ Cita Rosita Sigit Prakoeswa, ${ }^{1}$ \\ Shinzo Izumi, ${ }^{2}$ Malcolm S. Duthie, ${ }^{3}$ \\ Indropo Agusni ${ }^{1}$ \\ ${ }^{1}$ Department of Dermatology and \\ Venereology, Universitas Airlangga, \\ Dr. Soetomo Teaching Hospital, \\ Surabaya, Indonesia; ${ }^{2}$ Institute of \\ Tropical Disease, Universitas Airlangga, \\ Surabaya, Indonesia; ${ }^{3}$ Infectious Disease \\ Research Institute, Seattle, USA
}

\begin{abstract}
Leprosy is caused by Mycobacterium leprae infection and remains a major public health problem in many areas of the world. The Leprosy IDRI Diagnostic (LID)-1 antigen has demonstrated potential to improve the diagnostic capacity of local health centers and aid the development of strategies for the eventual control and elimination of leprosy from endemic areas. The diagnostic capacity of LID-1 has not yet, however, been studied in Indonesia. Objective: To determine the proportion of Indonesian leprosy patients that have circulating antibodies against LID-1. Sera from thirtyfive leprosy patients from Surabaya, Indonesia were evaluated using an Enzyme Linked Immunosorbent Assay (ELISA) detecting IgG antibodies against the LID-1 antigen. Anti-LID-1 antibody levels correlated with both the clinical form of leprosy and the bacterial index (BI). LID-1-specific IgG responses were higher in multibacillary (MB) than in paucibacillary (PB) leprosy patients. Our data indicate that the detection and measurement of serum IgG against LID-1 could be an effective tool for use in control programs in various states and municipalities in Indonesia.
\end{abstract}

\section{Introduction}

Leprosy is caused by Mycobacterium leprae infection and remains a major public health problem in many areas of the world. On a per country basis, Indonesia reports the third highest number of new cases of leprosy each year in the world, behind only India and Brazil. ${ }^{1}$ The disease is insidious and very difficult to reliably diagnose by simple clinical examination, especially in the earlier stages of $M$. leprae infection. Thus, timely diagnosis of leprosy remains a significant challenge and this commonly results in delay in treatment, and this in turn is usually associated with severe disability.

Although the detection of antibodies against the phenolic glycolipid (PGL)-I antigen has been reported as an auxiliary diagnostic tool and it has been indicated that serological examinations can be more sensitive than skin slit smears in diagnosing either multibacillary (MB) patients $(85.9 \%$ and $59.8 \%$ ) or paucibacillary (PB) leprosy patients $(75.4 \%$ and $1.8 \%),{ }^{2}$ no serological assays are currently in routine use to support the diagnosis of leprosy.

Leprosy Infectious Disease Research Institute (IDRI) Diagnostic (LID)-1 is a recombinant protein antigen that has demonstrated the potential to improve diagnostic capacity at local health centers and aid the development of strategies for the eventual control and elimination of leprosy from endemic areas. ${ }^{3}$ Although antibodies against LID-1 have been reported in many leprosy endemic regions, despite its importance in the global leprosy situation, they have not previously been assessed among the Indonesian population. Therefore, in the current report, we determined the capacity of the LID-1 antigen to aid leprosy diagnosis in the highly endemic area of East Java, Indonesia. Our data validate previous findings and indicate the potential for use of the LID-1 antigen to detect antibodies in the circulation of leprosy patients in Indonesia.

\section{Materials and Methods}

\section{Ethical considerations}

The Ethical Committee of Dr. Soetomo General Hospital approved this study (192/Panke.KKE/III/2017). Convenience sampling was used, with evaluation of sera from thirty five clinically defined leprosy patients from Surabaya, East Java, Indonesia. Clinical form (8 PB and $27 \mathrm{MB}$ ) and bacterial index (BI) were recorded for each patient.

\section{Detection of LID-1-specific antibodies}

Anti-recombinant protein detection enzyme linked immunosorbent assays (ELISAs) were conducted by coating 96well microtiter plates (Polysorp ${ }^{\circledR}$, Nunc, Rochester, NY) with $1 \mu \mathrm{g} / \mathrm{ml}$ LID-1 protein in bicarbonate buffer overnight at $4{ }^{\circ} \mathrm{C}$. Plates were then blocked for one hour at room temperature with PBST with $1 \%$ BSA on a plate shaker. Serum diluted appropriately in $0.1 \%$ BSA was added to each well, and plates were incubated at room tempera-
Correspondence: Nanny Herwanto, Department of Dermatology and Venereology, Universitas Airlangga - DR Soetomo Teaching Hospital, Jl. Mayjen. Prof. Dr. Moestopo, No. 47, Airlangga, Gubeng, Surabaya, Jawa Timur, 60286, Indonesia.

Phone: +6281333290006 .

E-mail: nannyherwanto@gmail.com

Key words: antibody, leprosy, LID-1.

Acknowledgments: The authors would like to thank Netherlands Leprosy Relief for supporting this project and also Malcolm S. Duthie (Infectious Disease Research Institute, Seattle, USA) for his generous support of the antigen.

Contributions: NH, Reporting: NH, SI, Editing and Publication: NH, S, CRSP, MSD, IA.

Conflict of interest: There is no potential conflict of interests in this study.

Received for publication: 1 February 2019. Accepted for publication: 6 February 2019.

This work is licensed under a Creative Commons Attribution-NonCommercial 4.0 International License (CC BY-NC 4.0).

(C) Copyright N. Herwanto et al., 2019 Licensee PAGEPress, Italy

Dermatology Reports 2019; 11(s1):8018 doi:10.4081/dr.2019.8018

ture for two hours with shaking. Plates were washed with buffer only, then horseradish peroxidase-conjugated IgG (Rockland Immunochemicals, Gilbertsville, PA), diluted in $0.1 \% \mathrm{BSA}$, added to each well and incubated at room temperature for one hour with shaking. After washing, plates were developed with peroxidase color substrate (Kirkegaard and Perry Laboratories, Gaithersburg, MD), and the reaction quenched by the addition of $1 \mathrm{~N}$ $\mathrm{H}_{2} \mathrm{SO}_{4}$. The optical density of each well was read at $450 \mathrm{~nm}$. Negative and positive responders were determined against a threshold value 0.031 OD unit, derived from the sum of the mean OD +2 times the standard deviation of a pool of sera from relatively healthy people.

\section{Results}

Figure 1 showed anti-LID-1 mean value of PB leprosy patients $(0.136 \pm 0.023$ OD unit) lower than observed in MB leprosy patients $(0.710 \pm 0.524 \mathrm{OD}$ unit). As seen in Figure 2A, 3 out of 5 PB leprosy patients $(60 \%)$ and 25 out of $27 \mathrm{MB}$ leprosy patients $(93 \%)$ had anti-LID antibody 
responses above a threshold determined using sera from non-leprosy individuals. Consistent with this, all serum samples from patients with a positive BI were positive (19 of $19 ; 100 \%)$, and even a majority of serum samples from patients with negative BI ( 9 of $13 ; 69 \%$ ), had antibodies against LID-1 (Figure 2B).

\section{Discussion}

Leprosy is a chronic infectious disease with a wide spectrum of clinical forms. Until now there has not been a gold standard diagnostic test for leprosy and its detection is mostly based on clinical signs and symptoms. ${ }^{4}$ Clinical classification based on World Health Organization guidelines places patients into one of two simplified categories: PB leprosy (smear negative, no nerves or only one nerve involved, maximum of five skin lesions) or MB leprosy (typically smear positive, having more than one nerve involved, more than five skin lesions). ${ }^{5}$

In endemic areas, sensitive and specific diagnostic laboratory tests would be of great use in aiding the detection of leprosy patients at an early stage of the disease. Serum antibodies against PGL-I, a specific $M$. leprae cell wall antigen, have however shown limited sensitivity in detecting PB leprosy patients. ${ }^{6}$

We assessed antibody responses against a chimeric fusion protein LID-1, comprising critical regions from the individual ML0405 and ML2331 proteins, in sera of Indonesia leprosy patients. Similar to the observations made when evaluating antiPGL-I responses, we found the highest levels of anti-LID-1 antibodies in MB patients, but lower or absent levels in PB patients. Thus, the IgG responses against each antigen positively correlated with the clinical forms and BI, but alternative approaches appear to be required for the reliable diagnosis of PB patients. These results do, however, further suggest that anti-protein antibody responses could be used to assist clinicians in determining the MDT regimen to provide to patients. ${ }^{7}$

In contrast to the IgM response that recognizes PGL-I (or its synthetic mimetic NDO-has), it is noteworthy that antibodies directed to the protein antigens are primarily of the IgG subclass. Since the generation of specific $\mathrm{IgG}$ responses typically requires T-cell help, it is likely that at certain stages of M. leprae infection, MB patients demonstrate specific T-cell reactivity to LID-1. Thus, the screening of strongly IgG-reactive antigens for T-cell reactivity may also rep- resent a diagnostic rationale to detect early stage MB leprosy. ${ }^{8}$

In this study, 3 out of 5 PB leprosy patients $(60 \%)$ and 25 out of 27 MB leprosy patients (93\%) showed anti-LID-1 seropositivity with mean value for PB leprosy pati- ents $0.136 \pm 0.023$ OD unit and for MB leprosy patients $0.710 \pm 0.524$ OD unit.

In PB leprosy patients, cellular immune responses control $M$. leprae replication and bacterial burden, and the humoral immune responses are restricted. This is demonstrat-

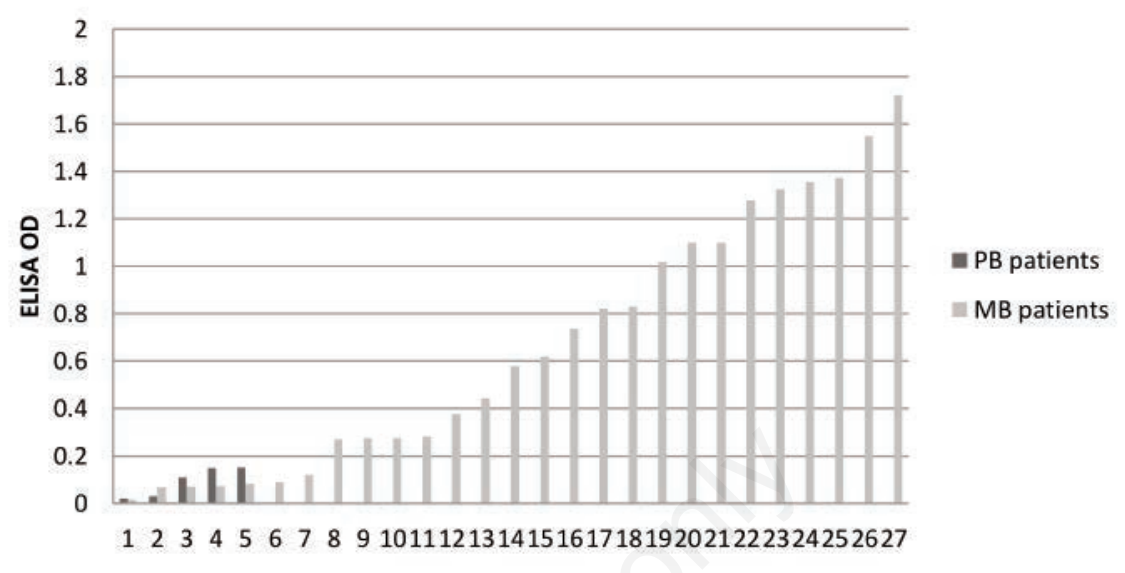

Figure 1. Anti-LID-1 value in PB and MB patients.
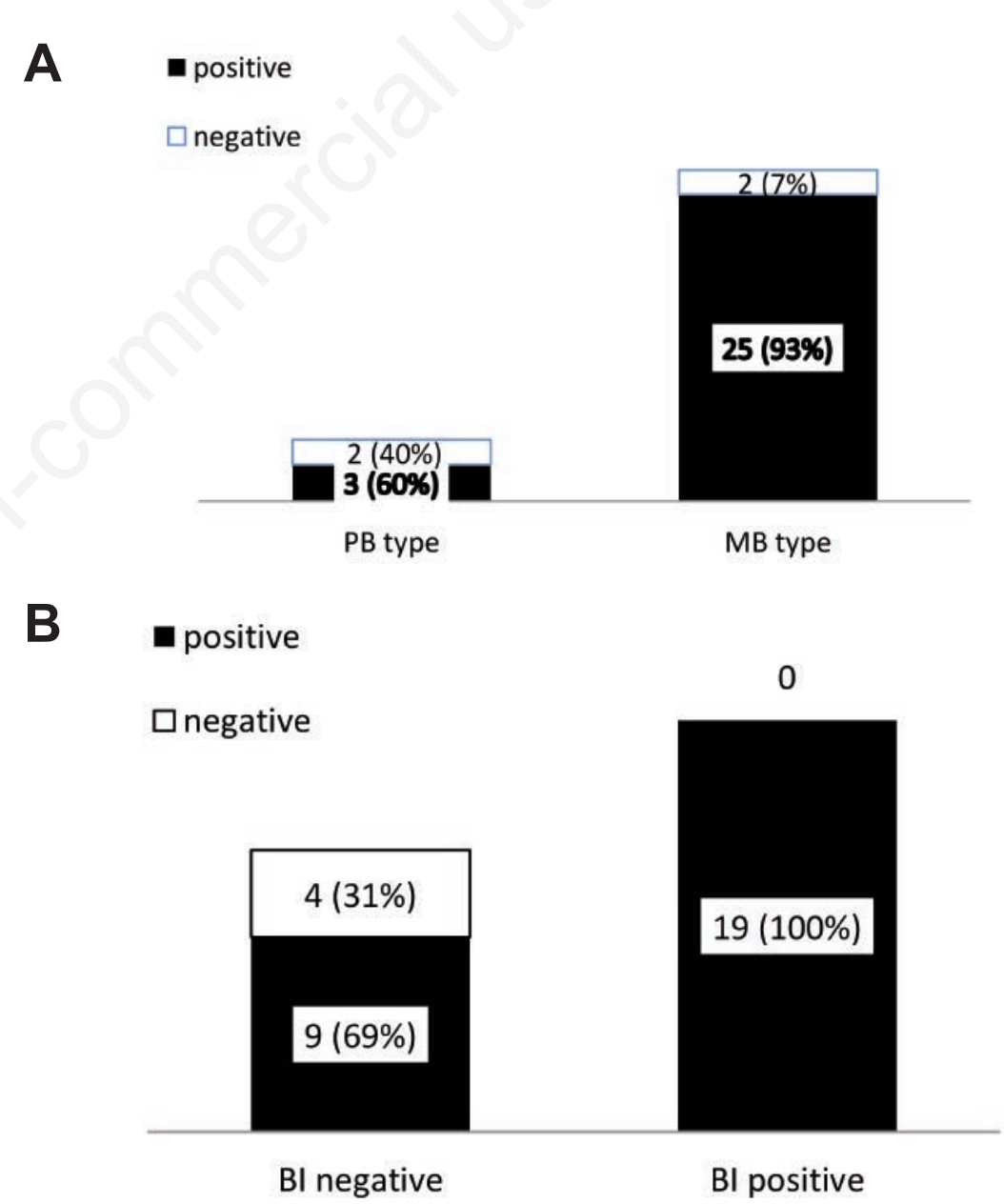

Figure 2. (A) Anti-LID-1 seropositivity in PB and MB leprosy patients; (B) Anti-LID-1 seropositivity in $\mathrm{BI}$ negative and $\mathrm{BI}$ positive leprosy patients. 
ed by the lower anti-LID-1 levels of PB leprosy patients, with anti-LID-1 mean value $(0.136 \pm 0.023$ OD unit) lower than observed in MB leprosy patients $(0.710 \pm 0.524$ OD unit). The lack of circulating antibodies to LID-1 in the majority of PB patients analyzed indicates that the antigen-specific antibodies are either not generated or do not persist, and should therefore not complicate the diagnosis of relapse or re-infection.

Surprisingly, our study showed higher seropositivity than previous studies. Silva et al. (2017) in Brazil found antibody LID-1 seropositivity in 25 out of 125 PB leprosy patients $(20 \%)$ and 41 out of 49 MB leprosy patients $(83.7 \%) .{ }^{6}$ Anti-LID-1 seropositivity was observed in 9 out of $13 \mathrm{BI}$ negative patients $(69 \%)$ with mean value $0.160 \pm 0.123$ OD unit and all 19 BI positive patients $(100 \%)$ with mean value $0.880 \pm 0.483$ OD unit. Amorim et al. (2016) also found a positive correlation between antibody LID-1 levels and BI $(r=0.84$. $\mathrm{p}<0.001){ }^{9}$

It is well established that the earlier leprosy is identified in a patient, the better their response to treatment. It stands to reason, then, that the earlier ineffective treatment can be identified, the earlier an adjustment can be made to render treatment effective to improve outcome. Our study validates the detection of antibodies against LID-1 as a strategy in Indonesian leprosy patients, further supporting the serodiagnostic potential of the LID-1 antigen.

\section{Conclusions}

Antigen specific antibody responses were highest in MB patients and lowest in PB patients. LID-1 antibody levels were related to the clinical form of leprosy as well as BI. In a leprosy endemic country like Indonesia, it is essential that new auxiliary techniques become available for disease control in various states and municipalities. Serological tests that do not require significant labor can detect asymptomatic M. leprae infection and may contribute to the control and eradication of leprosy.

\section{References}

1. World Health Organization. Global Leprosy Update 2015: Time for action, accountability and inclusion. Wkly Epidemiol Rec 2016;91:405-20.

2. Banerjee S, Biswas N, Kanti Das N, et al. Diagnosing leprosy: revisiting the role of the slit-skin smear with critical analysis of the applicability of polymerase chain reaction in diagnosis. International J Dermatol 2011;50:15227.

3. Duthie MS, Goto W, Ireton GC, et al. Use of protein antigens for early serological diagnosis of leprosy. Clin Vaccine Immunol 2007;14:1400-8.

4. Ridley DS. Classification of Leprosy
According to Immunity A Five-group System. Nature 2009;33:1215.

5. World Health Organization Expert Committee on Leprosy. Seventh Report. WHO Technical Report Series. Geneva: WHO; 1997;874.

6. Silva EA, Rosa PS, Belone AFF, et al. Serodiagnosis of leprosy and follow-up of household contacts using a commercial rapid test containing ND-O/LID-1 antigens. Leprosy Rev 2017;88:174-83.

7. Reece ST, Ireton G, Mohamath R, et al. ML0405 and ML2331 are antigens of Mycobacterium leprae with potential for diagnosis of leprosy. Clin Vaccine Immunol 2006;13:333-40.

8. Duthie MS, Hay MN, Rada EM, et al. Specific IgG antibody responses may be used to monitor leprosy treatment efficacy and as recurrence prognostic markers. Eur J Clin Microbiol Infect Dis 2011;30:1257-65.

9. Amorim FM, Nobre ML, Ferreira LC, et al. Identifying Leprosy and Those at Risk of Developing Leprosy by Detection of Antibodies against LID-1 and LID-NDO. PLoS Negl Trop Dis 2016;10. 